\title{
CAVITIES AT ATMOSPHERIC PRESSURE BEHIND TWO-DIMENSIONAL BODIES AT AN ANGLE OF ATTACK
}

\author{
P. M. HAESE ${ }^{\mathbf{I}}$
}

(Received 15 July, 2004; revised 14 February, 2005)

\begin{abstract}
This paper presents an interior source method for the calculation of semi-infinite cavities behind two-dimensional bluff bodies placed at an angle of attack in a uniform stream. Aspects under consideration include the pressure distribution along the body, especially just ahead of the separation point, lift and drag forces, and how these quantities vary with the angle of attack. We include discussion of the physical conditions of separation, and identify critical angles of attack for which the cavitating flow past an airfoil may (a) become unstable, or (b) yield the greatest lift to drag ratio.
\end{abstract}

\section{Introduction}

When a solid object is placed in a uniform stream of an incompressible liquid, a free surface sometimes springs from its edges, enclosing a cavity containing vapour. The surface of this cavity remains well defined for a certain distance aft of the body, before collapsing in an unsteady, turbulent wake. This whole process is known as "supercavitation". There is a constant pressure along the entire length of the cavity boundary, which in reality is lower than atmospheric pressure, resulting in collapse of the cavity at some point a finite distance downstream. However, we consider in the present paper only infinite-length cavities whose pressure is equal to the atmospheric pressure at infinity.

Historically, linearised theory has been very important in supercavitation research, arising from the assumption that the supercavitating body is very thin and makes a very small perturbation to the main stream fluid velocity. The thin-body theory of Tulin [17-19] and Geurst [6] paved the way for two-dimensional research, while other authors such as Grigoryan [8], Cumberbatch and Wu [5], Yakimov [20] and

\footnotetext{
${ }^{1}$ School of Applied Mathematics, The University of Adelaide, Adelaide SA 5005, Australia; e-mail: phaese@maths.adelaide.edu.au.

(C) Australian Mathematical Society 2005, Serial-fee code 1446-181 1/05
} 
Chou [4] considered three-dimensional linearised theory which is still prominent in research today. Even the most recent authors such as Kirschner [13], Nesteruk [14] and Semenenko and Semenenko [15] devote time to slender bodies.

However, while thin- and slender-body solutions provide good approximations to many aerodynamic and hydrodynamic problems, in practice all bodies have finite widths. We therefore need to account for body thickness, which introduces a nonlinear aspect to the problem. Complex-variable methods, such as the Schwartz-Christoffel mapping, are often used for general two-dimensional problems. These methods were employed extensively by authors such as Tulin [17] and Gurevich [9], but although they provide analytic solutions for many two-dimensional bodies, complex-variable methods are extremely difficult to extend into three dimensions, and this has only been done by a few authors such as Kaplan [11].

Of particular interest to us here is "smooth" separation, which is when the fluid separates from the body with finite curvature, and this corresponds to a zero pressure gradient on the body at separation. If actual separation were to occur before the point of smooth separation and if the body continues beyond the separation point, then the cavity streamline would pass into the body, which is physically unacceptable by the first part of the Brillouin condition (Gilbarg [7, page 322]). If actual separation occurred after the smooth separation point, the streamline would spring away from the body with infinite curvature, and there would be an infinite positive gradient in the pressure at the separation point. The pressure on the body at points ahead of the separation point would then be lower than that at the separation point, requiring the velocity on the body to exceed that on the cavity streamline. This then violates the second part of the Brillouin condition, which states that the maximum flow speed is achieved on the free boundary.

Now in reality, there is always a viscous boundary layer ahead of the separation point, so the actual flow near detachment is complicated, and may not necessarily be accurately represented by a potential model. This problem was noted and briefly discussed by Tulin in Streeter's Handbook [16], and is a subject of much debate. However, we do not consider viscosity here, and assume the smooth separation point to be the best approximation to separation in reality. Smooth separation in the thinbody limit was discussed by Haese [10], drawing several conclusions regarding smooth separation.

In the present article, we consider an alternative method for the solution of twodimensional asymmetric cavity flows, which can be readily extended into three dimensions. We consider supercavitation due to flow past two-dimensional asymmetric bodies, although we assume the cavity to continue all the way to infinity rather than collapsing, because at present there is no realistic model for asymmetric cavity closure. The cavitation number, which is proportional to the difference between the atmospheric pressure and the cavity pressure, is therefore zero for these cavities. We 
apply an interior source method in which a large number of line sources are placed inside the body and cavity. We consider two body shapes: ellipses and an airfoil-like body, from which we expect smooth separation from the nose region in both cases.

\section{Flow and boundary conditions}

We demand irrotational flow of an inviscid, incompressible fluid past the body, so a velocity potential exists and the flow is governed by Laplace's equation. We assume the velocity potential to be of the form

$$
\phi=U x+\Phi,
$$

where $\Phi$ is a (not necessarily small) perturbation to the uniform stream.

The velocity components in the $x$ - and $y$-directions due to this potential are, respectively,

$$
u=U+\Phi_{x}, \quad v=\Phi_{y} .
$$

We assume that the body touches the origin, and we define the $y$-coordinate of the body's surface as a function of the arclength, $s$, around its surface, so $y=f^{ \pm}(s)$ defines the top and bottom sides of the body.

The arclength $s$ satisfies

$$
d s^{2}=d x^{2}+d y^{2},
$$

with $s=0$ corresponding to the leading edge of the body when at zero angle of attack.

Fluid is not allowed to pass through the boundary of the body or cavity, so we enforce the kinematic boundary condition $n \cdot \nabla \phi=0$, where the normal to the surface is $n \propto \nabla\left(y-f^{ \pm}(s)\right)$. This leads to the exact boundary condition

$$
\Phi_{y}-f_{x}^{ \pm}(s)\left(U+\Phi_{x}\right)=0
$$

to be enforced at all points on both the top and bottom sides of the body and cavity.

Lastly, we require the pressure, $p$, on the cavity to be constant. This means that, given a fixed body from which the fluid separates, we need to guess a cavity shape, then alter it iteratively until constant pressure is achieved along its length. From the Bernoulli equation, the pressure is given by

$$
p=p_{A}+\frac{1}{2} \rho\left(U^{2}-u^{2}-v^{2}\right),
$$

where $u=U, v=0$ and $p=p_{A}$, the atmospheric pressure, at infinity. Without loss of generality, we choose $p_{A}=0$. 
We non-dimensionalise the pressure $p$ by reference to the quantity $\rho U^{2} / 2$, and all velocity components are likewise scaled by the free-stream speed $U$. The nondimensional pressure can then be written as

$$
\hat{p}=1-\hat{u}^{2}-\hat{v}^{2}
$$

where $\hat{u}$ and $\hat{v}$ are the non-dimensional velocity components, and $\hat{p}$ is in fact the same as the pressure coefficient

$$
C_{p}=\frac{p-p_{A}}{\rho U^{2} / 2}
$$

\section{Interior source method}

In order to find the appropriate perturbation velocity potential $\Phi$, we place a large number of sources within the body and cavity region, such that the stagnation streamline they generate matches the shape of the body and cavity boundary, as shown in Figure 1. Such a method has been employed in a number of other contexts, by authors such as Cao et al. [3] and Beck et al. [2].

3.1. Definition of nodes on the body and cavity We need to define a series of points on the body and cavity called "nodes", at which we will calculate the pressure. We assume that separation occurs at points with arclengths $s=s_{t}$ on the top and $s=s_{b}$ on the bottom, and that these points correspond to particular nodes on the body. We also guess an arclength $s=s_{0}$ that will define the stagnation point near the leading edge of the body (for a symmetric body, $s_{0}=0$ ). We use $N$ nodes on the top side of the body above the stagnation point, and a further $N$ nodes on the bottom side, making $2 N-1$ nodes in total. We set up several quadratic grids in order to cluster points near the three points of major interest, namely the stagnation point and the two separation points. These grids are symmetric about each of these three points of interest, to minimise any grid-scale oscillation. A small section of evenly-spaced grid is needed on the bottom side in asymmetric cases to fill in the gap between the grids coming from the stagnation point and the bottom separation point, as shown in Figure 2. The quadratically spaced grids are allowed to go downstream a distance about 100 times the arclength of separation on both the top and bottom sides.

We are now able to define the actual points on the body using the function $y=f^{ \pm}(s)$ and the relationship (2.3). For asymmetric bodies, the simplest way to do this is to calculate the body nodes at zero angle of attack, and then rotate them all by the desired angle of attack.

Initially, we guess a cavity shape satisfying two requirements: (a) the slope of the cavity just after separation will be the same as the slope of the body just before it, and 


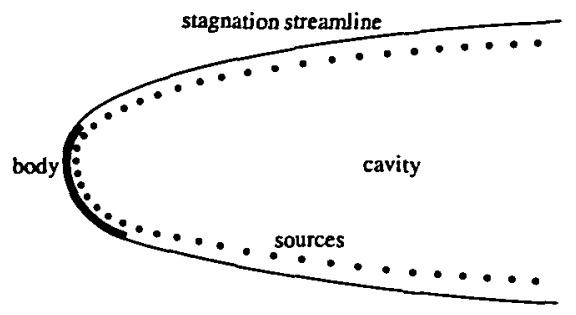

FIGURE 1. Placement of sources for an interior source method. The stagnation streamline generated by the sources matches the shape of the body and cavity.

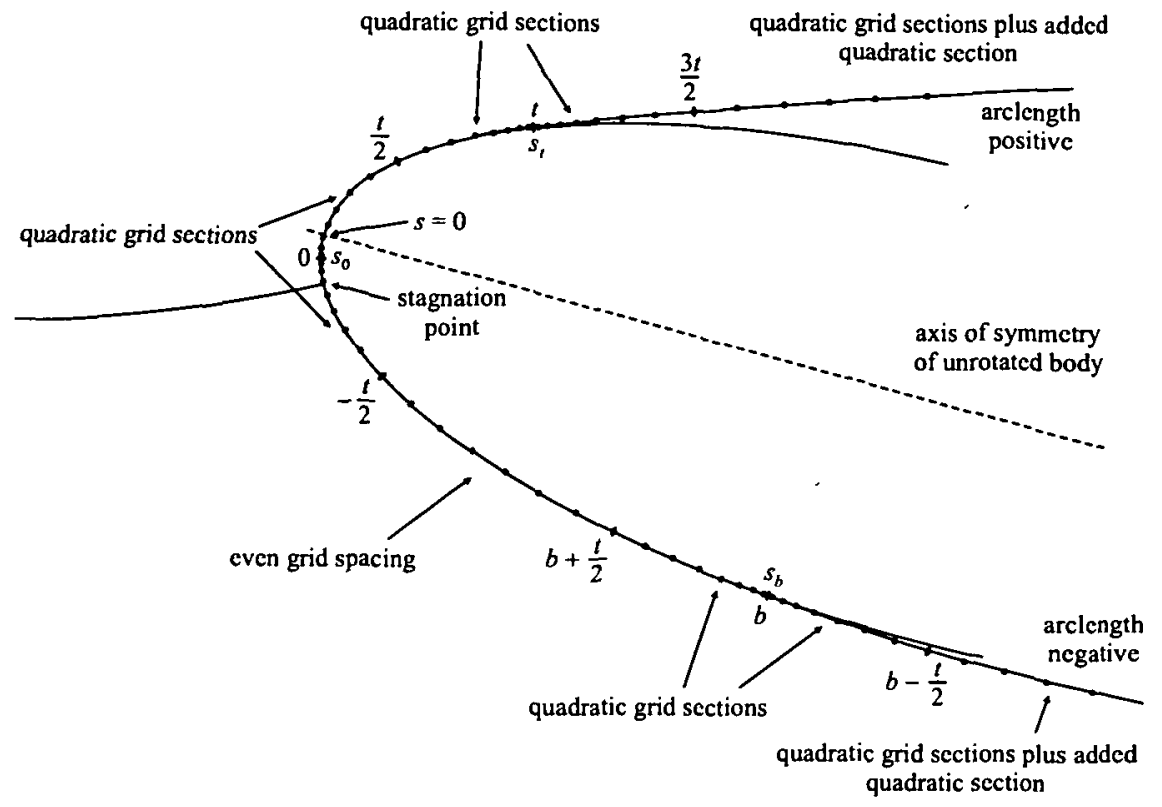

FIGURE 2. Example grid for a symmetric supercavitating body at an angle of attack. Arclength $s=0$ corresponds to the leading edge of the unrotated body. Node 0 is placed as close as possible to the new stagnation point of the body.
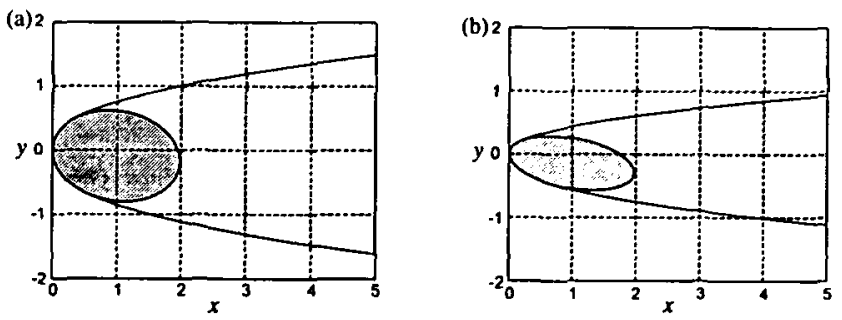

FIGURE 3. Cavity shapes behind ellipses of half-widths (a) 0.7 and (b) 0.4 at an angle of attack of $10^{\circ}$. The smooth separation points on the bottom sides are aft of those on the top sides. 
(b) far downstream, the cavity will behave like a parabola, as suggested by thin-body theory. The method for revising the cavity shape to obtain a constant pressure cavity is discussed in Section 3.3.

3.2. Placement and strength of sources In order to place the sources, we consider groups of three adjacent nodes. If the nodes are not collinear, then there exists a circle which passes through all three points, and we place the source along the radius from the middle of the three nodes to the centre of that circle. If the body or cavity is concave, then we will need to move in the opposite direction to ensure the source is placed within the body.

We place the source within the body at a distance equal to the local grid spacing. There is a trade-off here: the closer the sources are placed to the body, the less accurate the solution will be for the flow between the nodes, but the further we move inside the body, the more grid-scale oscillations we will observe when we calculate pressure values.

The velocity potential for the flow of a uniform stream of velocity $U$ past a set of $2 N-1$ sources is

$$
\phi=U x+\Phi=U x+\sum_{1-N}^{N-1} m_{j} \log \sqrt{\left(x-X_{j}\right)^{2}+\left(y-Y_{j}\right)^{2}},
$$

where $2 \pi m_{j}$ are the source strengths which are to be determined. Applying the boundary condition (2.4), we determine that

$$
\sum_{j=1-N}^{N-1} m_{j}\left[\frac{y_{i}-Y_{j}}{\left(x_{i}-X_{j}\right)^{2}+\left(y_{i}-Y_{j}\right)^{2}}-f_{i}^{\prime} \frac{x_{i}-X_{j}}{\left(x_{i}-X_{j}\right)^{2}+\left(y_{i}-Y_{j}\right)^{2}}\right]=U f_{i}^{\prime}
$$

for each node $i=1-N, \ldots, N-1$, where, given the shape of the body, the only unknowns in the equation are the $m_{j}$. This system of $2 N-1$ equations in $2 N-1$ unknowns must be inverted to determine the source strengths $2 \pi m_{j}$. Because the matrix is not sparse and has no special simplifying features that can be exploited, the linear matrix equation (3.2) must be solved by a full row reduction algorithm, which is computationally intensive.

Having determined the source strengths, we may then calculate the $x$ - and $y$ velocities at each node, and hence the pressure at each node using the Bernoulli equation (2.5).

\subsection{Modelling the cavity shape to achieve constant pressure When modelling} the cavity shape, it is important to incorporate several features of the flow:

- At the separation point, we need the slope of the cavity to remain finite and equal to that of the body on separation. To account for the physical constraints of 
the Brillouin condition (Gilbarg [7, page 322]), we require either smooth separation with finite curvature or non-smooth separation from the body's trailing edge. We also require the flow to separate from the body such that $y$ is locally a smooth function of $\left(s-s_{t}\right)^{1 / 2}$.

- We need to be able to modify the cavity shape iteratively so that the pressure is forced to be constant along its entire length.

- From the thin-body theory of Tulin [18], we know that the stagnation streamline for infinite-length cavities is parabolic far downstream. This can be shown to be true for non-thin bodies also. For example, if $y \sim x^{n}$ and $n<1 / 2$, then the resistance is zero, and if $n>1 / 2$, the resistance is infinite; only for $n=1 / 2$ is the resistance finite.

Since the cavity is semi-infinite in length, the pressure everywhere along the body must be equal to the atmospheric pressure at infinity, that is, $\hat{p}=0$. We use a small number of shifting nodes, which are nodes on the cavity at which we demand zero pressure. This is achieved using Newton's method to iteratively move the shifting nodes so that their pressures will all be zero. If the magnitude of the pressure at the shifting nodes is less than $10^{-4}$, then the pressure at the other cavity nodes should also be close to zero, providing we are using a suitable interpolation. Hence, for the other cavity nodes we satisfy the kinematic boundary condition, but do not force the pressure to take any particular value. We use this method in preference to enforcing zero pressure at all nodes along the cavity, because shifting all of the nodes along the cavity at once is both computationally intensive and numerically unstable. However, we choose the shifting nodes so as to minimise the variation in pressure along the cavity, so the solution is quite accurate. We can also vary the choice of shifting nodes to verify that the solution by this method is convergent.

The iterative scheme calculates the $y$-coordinates of the shifting nodes, but then the $y$-coordinates of the other nodes (that is, those between the shifting nodes) must be determined by interpolation. These values are calculated using cubic splines in a transformed plane: we define the variable $r_{t}=\sqrt{s-s_{t}}$ for the top side (and similarly for the bottom side) and construct a cubic spline in this variable, $r_{t}$, between each shifting node.

We require the slope of the cavity at the separation point to be finite and equal to that of the body, and since the spline passes through the last node on the body and the first shifting node on the cavity, we have thus fully determined the first spline. Splines further downstream are then determined by ensuring continuity in $r, r^{\prime}$ and $r^{\prime \prime}$ at each shifting node.

The final spline presents difficulties, since we have only a finite number of sources but the cavity behaves like a parabola downstream. This means that the source strengths for the final cavity nodes will not tend to zero as we would expect if the line of sources went on to infinity, but rather will tend to a non-zero value, in order to compensate for the truncation of the line of sources. Therefore, for the last cubic 
spline, instead of ensuring continuity in $y$ at the final node to define our spline, we choose the slope at the final node such that the pressure variation over the final nodes is minimised.

Most importantly, we note that the required features of the flow are satisfied by this choice of spline. In particular, the behaviour of the cavity close to the separation point, and the parabolic behaviour downstream, are captured. This is why the solution is accurate at all nodes, not just the shifting nodes.

3.4. Lift and drag coefficients Since we are dealing with infinitely long cavities, the drag is simply

$$
D=\int_{\text {body }} p d y \approx \frac{1}{2} \sum_{b+1}^{1}\left(p_{j}+p_{j-1}\right)\left(y_{j}-y_{j-1}\right) .
$$

The lift force on the body acts to push the body upwards, and since it is perpendicular to the drag force,

$$
L=-\int_{\mathrm{body}} p d x \approx-\frac{1}{2} \sum_{b+1}^{1}\left(p_{j}+p_{j-1}\right)\left(x_{j}-x_{j-1}\right) .
$$

\section{Results for elliptic bodies}

We consider a family of ellipses of the form

$$
\left(\frac{y}{a}\right)^{2}+(x-1)^{2}=1,
$$

where $a$ is the half-width of the ellipse. When $a=1$, the body is a circular cylinder, which is unchanged under an angle of attack. We therefore consider here only the more interesting true ellipses $a=0.4,0.5,0.6$ and 0.7 . Solutions with smooth separation from both the top and bottom sides were found for angles of attack $0^{\circ}, 2.5^{\circ}, 5^{\circ}, 7.5^{\circ}$ and $10^{\circ}$.

The node at which separation occurs on the top side is chosen to be $t=60$ in every case. When the angle of attack is zero, 310 nodes are used on both the top and bottom sides. Ten shifting nodes are added symmetrically on the top and bottom sides of the cavity, including three shifting nodes immediately downstream of the separation point. When the angle of attack is increased, the total number of nodes is also increased, and it is found that convergence of the scheme is more rapid (and in some cases only possible) if the node numbers of the furthest downstream shifting nodes on the top side are greater than those on the bottom side. 


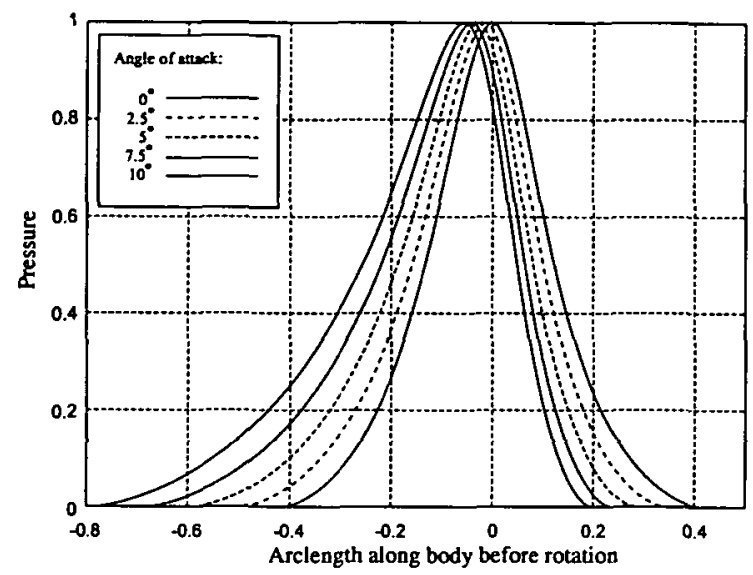

FIGURE 4. Body pressure distributions for the ellipse of half-width 0.4 at angles of attack $0^{\circ}, 2.5^{\circ}, 5^{\circ}$, $7.5^{\circ}$ and $10^{\circ}$. Positive arclength corresponds to the top side of the body before rotation.

Cavity shapes for the flow past ellipses with $a=0.7$ and 0.4 held at an angle of attack of $10^{\circ}$ are shown in Figure 3. As expected, separation occurs later on the bottom side than on the top side, and this effect is more pronounced for the thinner body. The stagnation points are at arclengths -0.1115 and -0.0567 respectively, so both are below the leading edge of the rotated bodies.

Figure 4 shows the body pressure distributions for the body of half-width 0.4 at various angles of attack. As the body is rotated, the smooth separation point on the top side shifts forward, but by decreasing amounts. The rear separation point shifts towards the back of the body, and the distance it shifts appears to be proportional to the angle of attack. The resulting effect is to have a broader pressure distribution on the bottom side of the body than on the top side, and this increase in pressure is responsible for the lift on the body.

Similar behaviour to that shown in Figure 4 is observed for elliptic bodies with half-widths $0.5,0.6$ and 0.7 . When the angle of attack is zero, the smooth separation points on the top and bottom sides have the same arclength. As the angle of attack is increased, the smooth separation point on the top side of the body shifts forward by a distance that appears independent of the body width. The rear separation point shifts towards the back of the body, but the distance it shifts is increased for the thinner ellipses.

The drag force for each case is shown in Figure 5. For thinner ellipses, the drag is smaller, since the body causes a smaller perturbation to the flow. As the angle of attack increases, the drag increases, because the effective chord of the bodies is made wider in the flow. The drag increases more for the case of the thinner ellipses, since the effective chords of these bodies grow more rapidly when the angle of attack is 
introduced.

Figure 6 shows the lift force for each ellipse half-width and each angle of attack. When the angle of attack is zero, the lift should be zero; the values calculated by the numerical method are at most $6 \times 10^{-6}$ for any of the ellipse half-widths considered, indicating that the error in the calculation is quite small. The lift decreases as the body thickness increases, which is reasonable since, as the thickness increases, we apprcach a circle, for which there is no lift no matter what the angle of attack.

The lift force increases approximately linearly with the angle of attack, which is not unreasonable since we know that for the case of a flat plate, the relationship is indeed linear. If $\bar{\alpha}$ is the angle of attack measured in radians, then the coefficient of lift when the bottom side of the thin flat plate is fully wetted is, from Tulin [17], $C_{L}=\pi \bar{\alpha} / 2$.

Now if we take the thin-body limit of an ellipse, it could be argued that we actually have a flat plate, so for an ellipse of length 2 with its bottom side fully wetted and its top side non-wetted (that is, with separation from the leading edge), we might expect the lift to be $L=\pi^{2} \alpha / 180$, where $\alpha$ is the angle of attack in degrees.

Now for this case $d L / d \alpha \approx 0.0548$, so we might expect that as the thickness of the ellipse approaches zero, the slope of the graph in Figure 7 would tend to this value. Clearly, this is not the case, however, and the slope, while positive, is much less than 0.0548 .

This difference may be explained because Tulin's thin-body theory relates to a plate which is fully wetted along its bottom edge. In taking the thin-body limit of the ellipse, although its shape tends to that of the flat plate, we know that it still has curvature. Therefore from Haese [10], smooth separation must occur from the ellipse at some point before the point of maximum thickness. The ellipse therefore cannot be fully wetted along its bottom edge, and the lift is lower than that for the case considered by Tulin.

Furthermore, although the smooth separation point for a thin body is not exactly at the nose, it occurs at a small value of arclength relative to the length of the body. Therefore, when a thin ellipse is rotated a small amount and the smooth separation point on the bottom side migrates backward, we still do not expect a great portion of the bottom side to be wetted, and a portion of the upper surface may still be wetted, reducing the lift further.

\section{Results for an airfoil at an angle of attack}

We consider a Joukowski-like airfoil with equation

$$
y=\varepsilon x^{1 / 2}(c-x)^{3 / 2},
$$

where $\varepsilon$ is a measure of the airfoil thickness and $c$ is the chord length of the airfoil. This airfoil has its maximum width at $x=c / 2$ and an inflexion point at $x=(1+\sqrt{3}) c / 4$, 


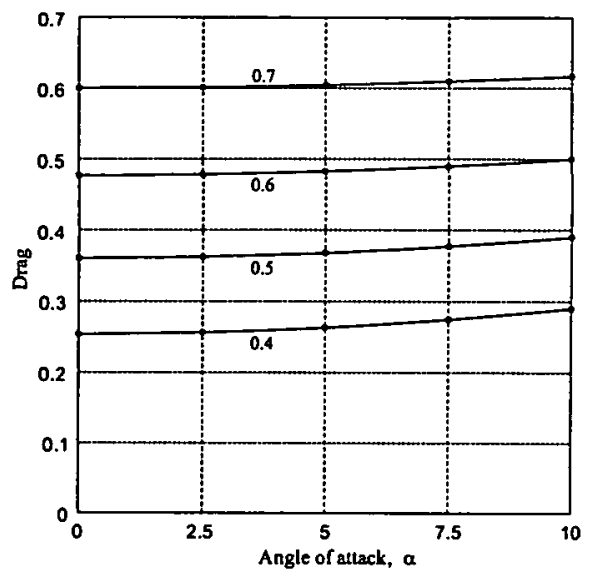

FIGURE 5. Drag forces on ellipses of half-widths $0.4,0.5,0.6$ and 0.7 at angles of attack of $0^{\circ}, 2.5^{\circ}, 5^{\circ}$, $7.5^{\circ}$ and $10^{\circ}$.

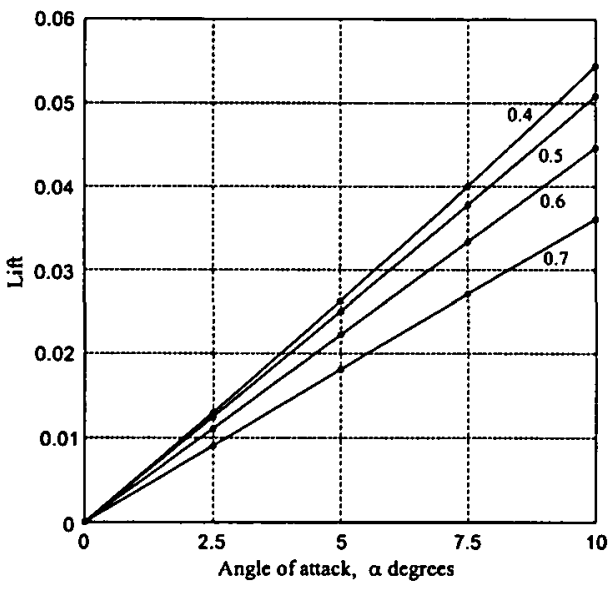

FIGURE 6. Lift forces on ellipses with half-widths $0.4,0.5,0.6$ and 0.7 at angles of attack of $0^{\circ}, 2.5^{\circ}, 5^{\circ}$, $7.5^{\circ}$ and $10^{\circ}$.
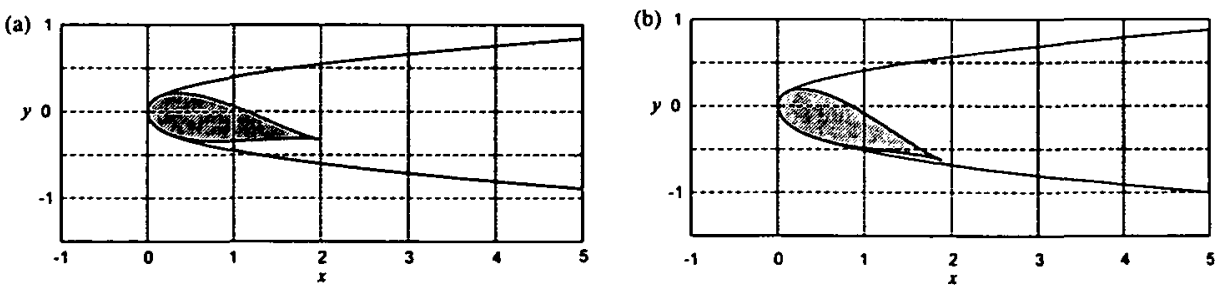

FIGURE 7. Cavity shapes behind an airfoil at angles of attack (a) $10^{\circ}$ and (b) $20^{\circ}$. The smooth separation points on the bottom sides are aft of those on the top side. 
after which the body is concave. The airfoil is cusped at its trailing edge. Here, we choose $\varepsilon=0.2$ and $c=2$.

Using the definition of arclength (2.3), we obtain

$$
\left(\frac{d y}{d s}\right)^{2}=\left(1+\frac{x^{2}(2-x)^{2}}{y^{2}(1-2 x)^{2}}\right)
$$

from which we may calculate the coordinates of the nodes on the body.

We expect that as the angle of attack on the airfoil increases, the smooth separation point on the bottom side will move back towards the trailing edge. When the angle of attack is sufficient, the smooth separation point will be pushed right back to the inflexion point. At higher angles of attack, any point of smooth separation would have to be on the concave region of the body, but this is geometrically infeasible since the cavity generated would intersect the body. Hence beyond a critical angle of attack where the smooth separation point lies at the point of inflexion, there will be no more smooth separation points on the bottom side of the body. The bottom side will be fully wetted, and non-smooth separation will occur at the trailing edge.

Figure 7 shows cavity shapes with smooth separation formed behind the airfoil at angles of attack of $10^{\circ}$ and $20^{\circ}$, and the results support the qualitative assessment above. The cavity shape on the top side of the body is very similar in each case.

In Figure 8 (a), we show the cavity shape for an angle of attack of $22.5^{\circ}$, and we observe that the cavity on the bottom side narrowly misses the trailing edge of the body. As the angle of attack is increased further, the cavity springing with smooth separation from the bottom side strikes the trailing edge, due to the concavity of the latter section of the body. This geometrically infeasible flow occurs despite the bottom-side smooth separation point being on the convex section of the body. An example of this situation is shown in Figure $8(\mathrm{~b})$, which is the flow for an angle of attack of $25^{\circ}$. In practice, the cavity would reattach itself to the body, before separating again non-smoothly at the trailing edge, although we do not model such cases here; see Kinnas and Fine [12] for examples.

We therefore determine that under the present model, the flow becomes geometrically infeasible before we reach the angle of attack for which the smooth separation point is at the inflexion point.

By the time the angle of attack reaches $26.25^{\circ}$, there is no smooth separation point on the convex region on the lower side of the body. This case is shown in Figure 8 (c), and as anticipated above, separation on the bottom side is then forced all the way back to the trailing edge. The flow is once again physically feasible, since the cavity does not cut the body.

The locations of smooth separation points on the top and bottom sides of the bodies are shown as functions of the angle of attack in Figure 9. In order to show these points on the one graph, we again use the magnitude of the smooth separation arclength. 

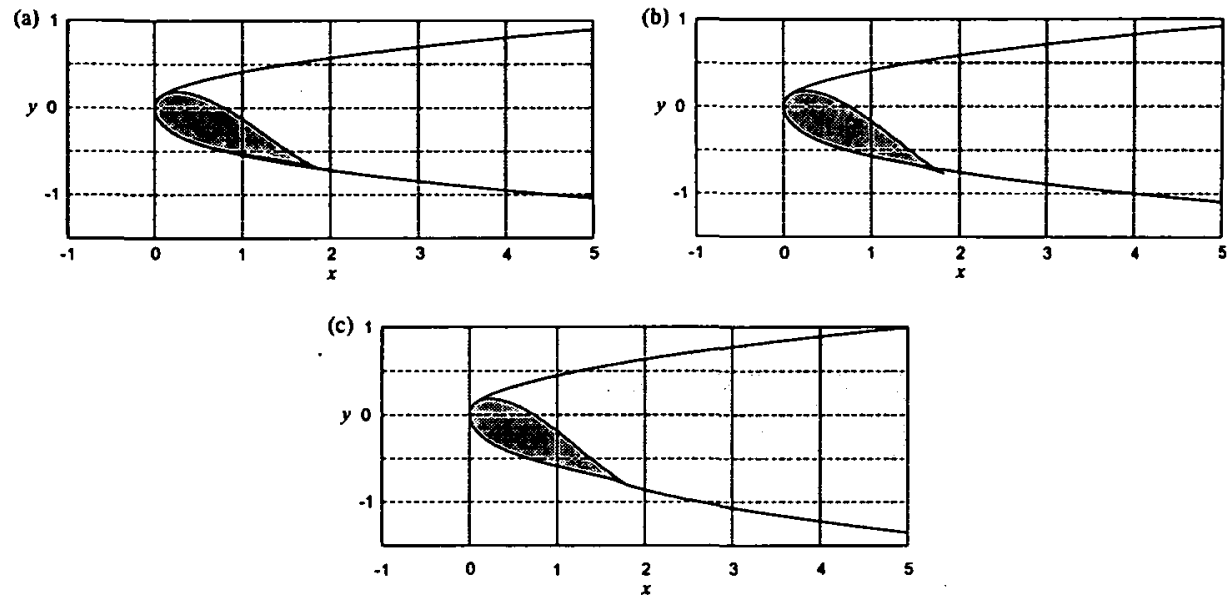

FIGURE 8. Cavity shapes behind an airfoil at angles of attack (a) $22.5^{\circ}$, (b) $25^{\circ}$ and (c) $26.25^{\circ}$. In case (a), the stagnation streamline from the separation point on the bottom side narrowly misses the trailing edge of the body, so this flow is feasible. As the angle of attack is increased, while a smooth separation point still exists on the convex region of the body, the cavity strikes the trailing edge. The flow in (b) is an example of such a case, and is physically infeasible. In (c), the angle of attack has been increased sufficiently so that there is no smooth separation point on the bottom side of the body, and in this case non-smooth separation is observed from the trailing edge.

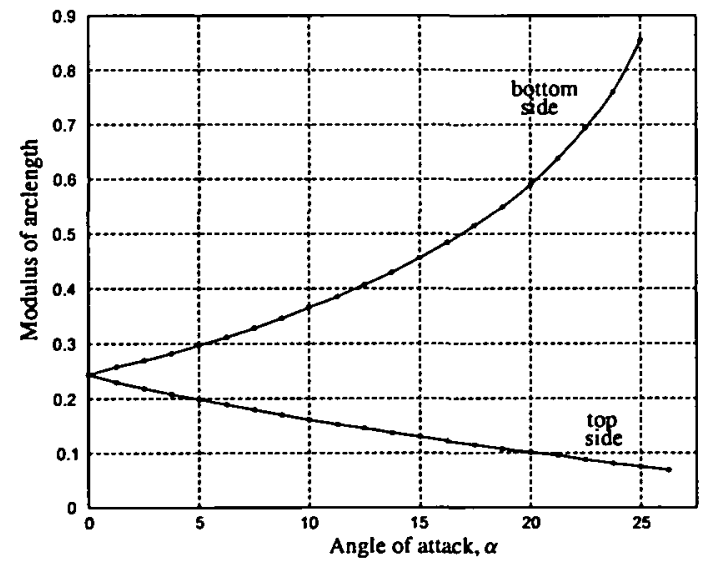

FIGURE 9. Arclengths of smooth separation on the top and bottom sides for an airfoil at various angles of attack. The modulus of the arclength is shown in each case. When the angle of attack reaches $26.25^{\circ}$, there is no longer a smooth separation point on the bottom side of the body, and non-smooth separation occurs at the trailing edge. 
When the angle of attack is zero, the smooth separation points on the top and bottom sides are at the same arclength, as expected. As the angle of attack increases, the smooth separation point on the top side slowly moves forward toward the leading edge of the unrotated body, and the smooth separation point on the bottom side moves back increasingly rapidly. When the angle of attack is about $22.5^{\circ}$, the flow becomes infeasible since the cavity generated strikes the trailing edge of the body. When the angle of attack reaches $26.25^{\circ}$, however, the flow becomes physically feasible again, but there are no longer smooth separation points on the bottom side. The separation point on the top side keeps moving forward toward the leading edge.

The body pressure distributions for angles of attack of $0^{\circ}, 5^{\circ}, 10^{\circ}, 15^{\circ}$ and $20^{\circ}$ are shown in Figure 10, and are qualitatively similar to those for an ellipse at an angle of attack: the distribution is symmetric when the angle of attack is zero, and as the angle of attack increases, the pressure distribution becomes skewed toward the top side of the body. The pressure distribution is broader on the bottom side of the body than on the top side, and this is responsible for the lift force.

Figure 11 shows the body pressure distribution when the angle of attack is $26.25^{\circ}$. Separation on the top side is smooth, the pressure dropping steadily down to the atmospheric pressure with zero gradient at the separation point; the scale of the figure obscures this highly localised feature. On the bottom side, the body is fully wetted, with non-smooth separation occurring at the trailing edge. The pressure drops while on the convex section of the body, but increases when the body shape becomes concave, since the flow is once again forced to deviate away from the uniform stream. Finally, the pressure drops to atmospheric pressure with infinite gradient at the trailing edge.

The lift and drag forces acting on the airfoil are shown in Figure 12. As the angle of attack increases, so too does the drag on the body, in agreement with the calculations for the ellipse. The lift force on the body increases linearly for small angles of attack, in agreement with Batchelor [1, page 446], although Batchelor assumed the body to be fully wetted on the bottom side. For larger angles of attack, the smooth separation point on the bottom side moves backward more rapidly, and this causes an increase in the rate of change of lift, $d L / d \alpha$.

When there is no longer a smooth separation point on the convex region on the bottom side of the body, the separation point jumps to the trailing edge. Since the effective chord of the body in the flow is suddenly increased, the drag force jumps as shown by the dashed line. A corresponding jump in the lift force is also observed, since the bottom side of the body is now fully wetted and lift is generated along its entire length. In this case, the flow resembles more closely the lifting-body problems considered by authors such as Tulin [17] and Batchelor [1].

The lift advantage obtained by forcing separation on the bottom side of the body back to the trailing edge is quite large, and highly desirable for lifting airfoils. We expect that for thinner bodies, the critical angle of attack at which this occurs will be 


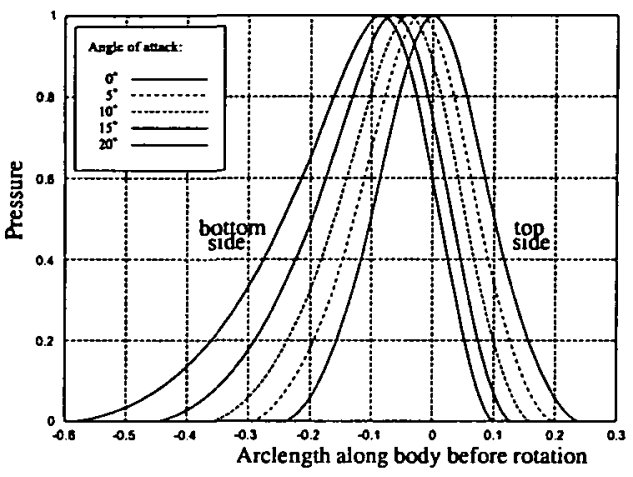

FIGURE 10. Body pressure distributions for an airfoil at angles of attack $0^{\circ}, 5^{\circ}, 10^{\circ}, 15^{\circ}$ and $20^{\circ}$. The positive arclength corresponds to the top side of the body before rotation.

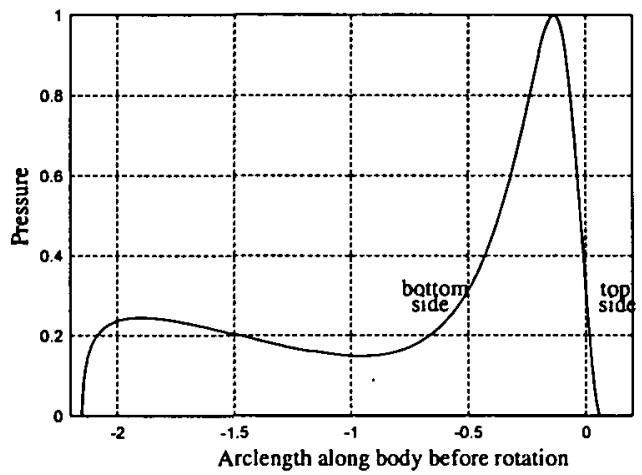

FIGURE 11. Body pressure distributions for an airfoil at an angle of attack $26.25^{\circ}$. Separation on the top side is smooth, but on the bottom side separation occurs non-smoothly with infinite pressure gradient at the trailing edge.

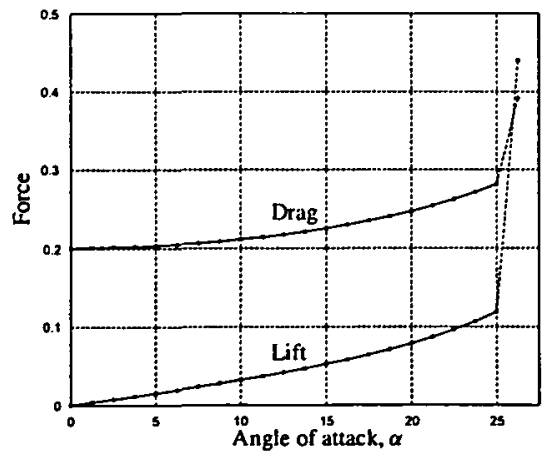

FIGURE 12. Drag and lift forces on an airfoil at various angles of attack. The dashed lines show the jumps in the drag and lift when the bottom side smooth separation point is forced back to the trailing edge of the body. 
reduced, and given that the drag decreases as the body thickness decreases, thinner airfoils carry several advantages.

\section{Conclusion}

The method presented in this paper enables the calculation of infinite-length cavities behind two-dimensional asymmetric bodies, and incorporates important physical conditions in the flow such as the Brillouin condition (Gilbarg [7, page 322]) for smooth separation.

Thus far, however, we have made no mention of the finite-length (non-zero cavitation number) case. This case is significant because, in practice, cavities do not extend to infinity, but rather collapse at some point downstream in a turbulent wake. A model for asymmetric cavitation closure is not presented here, however, because the shape and nature of this closure is uncertain.

\section{Acknowledgement}

The author would like to sincerely thank Professor Ernie Tuck of the University of Adelaide for his continual support and guidance throughout this project.

\section{References}

[1] G. I. Batchelor, An introduction to fluid dynamics (Cambridge University Press, London, 1967).

[2] R. F. Beck, Y. Cao and T.-H. Lee, "Fully nonlinear water wave computations using the desingularized method", in 6th International Conference on Numerical Ship Hydrodynamics, lowa, (1993).

[3] Y. Cao, W. W. Schultz and R. F. Beck, "Three-dimensional desingularized boundary integral methods for potential problems", Internat. J. Numer. Methods Fluids 12 (1991) 785-803.

[4] Y. S. Chou, "Axisymmetric cavity flows past slender bodies of revolution", J. Hydronautics 8 (1974) 13-18.

[5] E. Cumberbatch and T. Y. Wu, "Cavity flow past a slender pointed hydrofoil", J. Fluid Mech. 11 (1961) 187-208.

[6] J. A. Geurst and R. Timman, "Linearized theory of two-dimensional cavitational flow around a wing section", in IX International Congress of Applied Mathematics, (1956).

[7] D. Gilbarg, "Jets and cavities", in Handbuchder Physik (ed. S. Flügge), (Springer, Berlin, 1960) 9.

[8] S. S. Grigoryan, "An approximate solution of the problem of separated flow past an axially symmetric body", Prikl. Mat. Meh. 23 (1959) 951-953 (Russian); translated as J. Appl. Math. Mech. 23 (1959) 1351-1355.

[9] M. I. Gurevich, Theory of jets in ideal fluids (Academic Press, New York, 1965).

[10] P. M. Haese, "Linearised cavity theory with smooth detachment", Aust. Math. Soc. Gaz. 28.4 (Oct 2001 ) 187-193. 
[11] C. Kaplan, "On a new method for calculating the potential flow past a body of revolution", NACA Technical Report 752, 1943.

[12] S. A. Kinnas and N. E. Fine, "A numerical nonlinear analysis of the flow around two- and threedimensional partially cavitating hydrofoils", J. Fluid Mech. 254 (1993) 151-181.

[13] I. N. Kirschner, Numerical modelling of supercavitating flows (von Karman Institute for Fluid Dynamics, Belgium, 2001).

[14] I. Nesteruk, "Influence of the flow unsteadiness, compressibility and capillarity on long axisymmetric cavities", in Fifth International Symposium on Cavitation (Osaka, Japan, November 2003), (2003).

[15] V. N. Semenenko and T. N. Semenenko, "Prediction of the unsteady cavities in cascades", in Fifth International Symposium on Cavitation (Osaka, Japan, November 2003), (2003).

[16] V. L. Streeter, Handbook of fluid dynamics (McGraw Hill, New York, 1961).

[17] M. P. Tulin, "Supercavitating flow past foils and struts", in Proceedings of the NPL Symposium on Cavitation in Hydrodynamics, (1955).

[18] M. P. Tulin, "Steady two-dimensional cavity flows about slender bodies", David Taylor Model Basin Report 834, May 1953.

[19] M. P. Tulin and P. Birkart, "Linearized theory for flows about lifting foils at zero cavitation number", David Taylor Model Basin Report C-638, Feb 1955.

[20] J. Yakimov, "About axisymmetric detached flow around a body of rotation at small cavitation number”, J. Appl. Math. Mech. 32 (1968) 3, (Russian). 